\title{
BROOKHIGEN
}

NATIONAL LABORATORY

BNL-98381-2012-IR

\section{FEA Simulations of Magnets with Grain Oriented Steel}

\author{
Holger Witte
}

August 2012

Physics Department

Brookhaven National Laboratory

\section{U.S. Department of Energy Office of Science}

Notice: This manuscript has been authored by employees of Brookhaven Science Associates, LLC under Contract No. DE-AC02-98CH10886 with the U.S. Department of Energy. The publisher by accepting the manuscript for publication acknowledges that the United States Government retains a non-exclusive, paid-up, irrevocable, world-wide license to publish or reproduce the published form of this manuscript, or allow others to do so, for United States Government purposes. 


\section{DISCLAIMER}

This report was prepared as an account of work sponsored by an agency of the United States Government. Neither the United States Government nor any agency thereof, nor any of their employees, nor any of their contractors, subcontractors, or their employees, makes any warranty, express or implied, or assumes any legal liability or responsibility for the accuracy, completeness, or any third party's use or the results of such use of any information, apparatus, product, or process disclosed, or represents that its use would not infringe privately owned rights. Reference herein to any specific commercial product, process, or service by trade name, trademark, manufacturer, or otherwise, does not necessarily constitute or imply its endorsement, recommendation, or favoring by the United States Government or any agency thereof or its contractors or subcontractors. The views and opinions of authors expressed herein do not necessarily state or reflect those of the United States Government or any agency thereof. 


\title{
FEA Simulations of Magnets with Grain Oriented Steel
}

\author{
Holger Witte*
}

August 6, 2012

\begin{abstract}
One of the potential successors of the Large Hadron Collider is a Muon Collider. Muons are short-lived particles, which therefore require fast acceleration. One potential avenue is a very fast cycling cyclotron, where the bending is supplied by a combination of fixed-field superconducting magnets and fast ramping normal conducting iron-cored coils.

Due to the high ramping rate (around $1 \mathrm{kHz}$ ) eddy current and hysteresis losses are a concern. One way to overcome these is by using grain-oriented soft-iron, which promises superior magnetic properties in the direction of the grains.

This note summarizes efforts to include the anisotropic material properties of grain-oriented steel in finite element analysis to predict the behaviour of the dipole magnets for this accelerator.

It was found that including anisotropic material properties has a detrimental effect on model convergence. During this study it was not possible to include grain oriented steel with an accuracy necessary to study the field quality of a dipole magnet.
\end{abstract}

\section{Introduction and Motivation}

One potential successor of the Large Hadron Collider is a Muon Collider $(1.5 \mathrm{TeV}$ or more). Muons are short-lived particles (around $2.2 \mu \mathrm{s}$ ), which therefore need to be accelerated quickly to their desired energy. A new type of synchrotron has been suggested [4], proposing to accelerate muons up to $750 \mathrm{GeV}$ in the Tevatron tunnel. The lattice of this synchrotron implies that the required bending of the particles is provided by a combination

\footnotetext{
${ }^{*}$ Brookhaven National Laboratory, Bldg. 901A, PO Box 5000, Upton, NY, 11973, USA. EMail: hwitte@bnl.gov
} 
of superconducting and normal conducting magnets (see Fig. 1 (a)). The 8 T superconducting magnets supply a DC field, which is complemented by normal conducting iron cored coils supplying a magnetic field of $\pm 1.8 \mathrm{~T}$. This allows to tune the integral bending power to keep the bending radius constant.
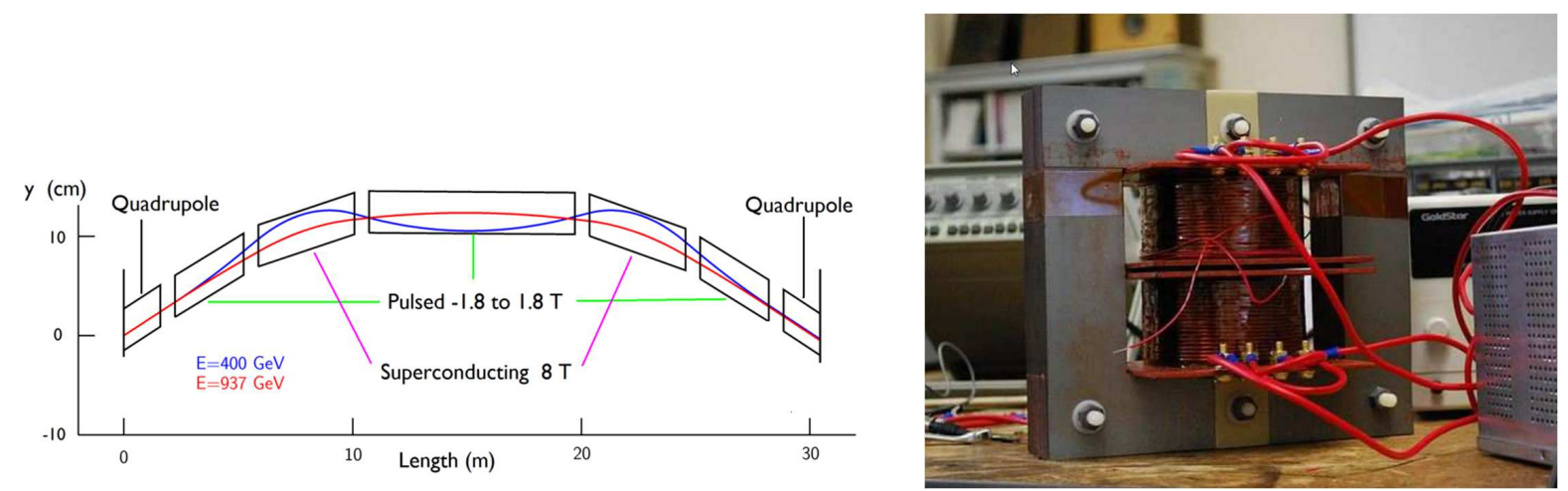

Figure 1: Lattice of the fast cycling synchrotron (a) and test dipole magnet to demonstrate feasibility (b). The gap of the test magnet is $46 \times 46 \times 1.5 \mathrm{~mm}$. The laminations were cut with wire EDM. The wound coils use a 12 gauge copper wire. The magnet is powered with a LC circuit with capacitor and IGBT switch (400V, $4.1 \mathrm{~J}$ and a peak current of $54 \mathrm{~A})$.

A drawback of this solution is that due to the rapid acceleration the iron-cored magnets need to be ramped equally fast, at a rate of about $1 \mathrm{kHz}$. This is higher by an order of magnitude in comparison to existing systems. A concern are eddy-current heating and magnetization losses. To overcome this it was suggested to use grain-oriented Si-steel, which possesses superior magnetic properties in the preferred direction.

A test magnet was built (see Fig. 1 (b)), but unfortunately it did not perform as expected. One potential source of error is inaccurate modelling of the highly anisotropic soft-iron. The purpose of this report is to explore possible ways how to include the anisotropic material properties in commercial finite element packages, to allow to identify potential sources of error and to optimize the present design.

\section{Simulation Details}

\subsection{Grain Oriented Steel}

Grain oriented steel available from a number of manufacturers, e.g. ThyssenKrupp (KurtSchumacher-Str. 95, D-45881 Gelsenkirchen, Germany), AK Steel (AK Steel Corporation, West Chester, OH 45069, USA) or Thomas \& Skinner (Indianapolis Offices, 1120 East 23rd St., Indianapolis, IN 46206, USA). The manufacturers supply limited information about their products, which indicate that the magnetic properties are extremely good if 
the magnetic field is aligned with the grain orientation. However, the properties deteriorate quickly with the angle to the grain direction.

Fig. 2 (a) shows an example from the company AK Steel for TRAN-COR H, which is commercially available Si-transformer steel. The figure illustrates that the permeability drops rapidly for angles larger than $10^{\circ}$ between applied magnetic field strength and grain orientation.
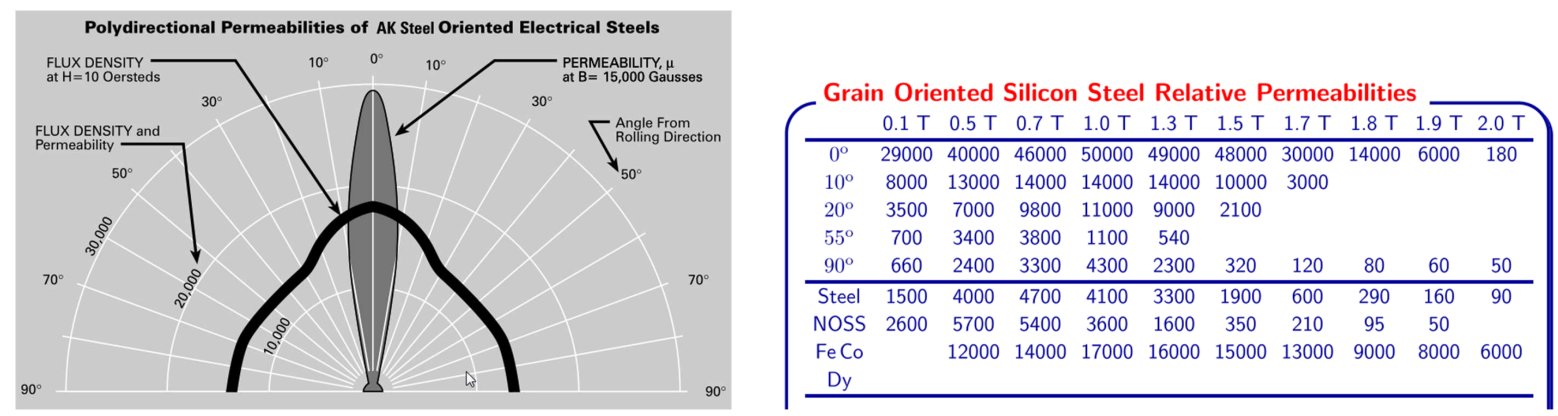

Figure 2: Guaranteed performance of grain oriented electrical steel from AK Steel (a) and measured magnetic permeability $\mu_{r}$ of used grain oriented steel.

To reconfirm the material properties given by the manufacturer grain oriented steel used in a test magnet was re-measured using an Epstein frame. The table in Fig. 2 (b) shows the measured values for various angles and fields (data from [3]). Fig.3 (a) and (b) show graphical representations of the data. Both figures highlight the strong anisotropic behaviour: the magnetic permeability can drop by a factor 10 or more for angles larger than $10^{\circ}$.

To predict the behaviour of iron cored magnets correctly, the data from table 2 (b) needs to be implemented into a finite element analysis.

\section{Simulation Details}

\subsection{COMSOL}

COMSOL is a general purpose 2D/3D finite element code, which can address structural, thermal and electromagnetic problems. A prominent feature of Comsol is its capability to solve multiphysics problems, that is to include the various couplings between different application modes.

The 2D/3D application mode of Comsol in principle is capable of accepting a tensor for the magnetic permeability $\mu_{r}$ (either $2 \times 2$ or $3 \times 3$ ). In general any component (or tensor element) of $\mu_{r}$ can either be entered as a table (with interpolation) or as a function in MATLAB notation.

An advantage of COMSOL is that several different solvers/pre-conditioners are available, which can help to overcome convergence problems. Another powerful feature of COMSOL 

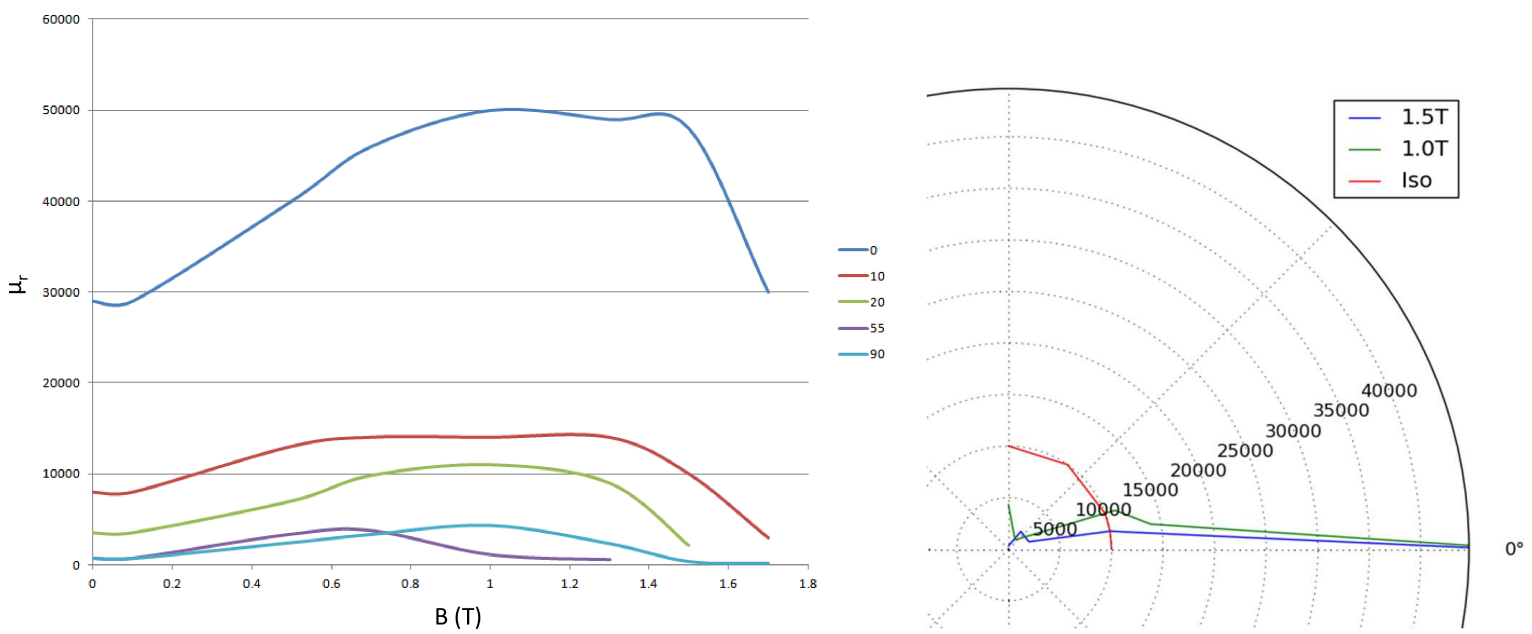

Figure 3: Magnetic permeability of grain oriented steel up to $1.7 \mathrm{~T}$ for various angles between the grain orientation and the applied field strength (a) and polar plot of the magnetic permeability of grain oriented steel for 1.0 and $1.5 \mathrm{~T}$ (b). For illustrative purposes isotropic material is also shown.

is that generic ODEs can be solved.

\subsection{VectorFields/Cobham Opera}

Opera is a $2 \mathrm{D} / 3 \mathrm{D}^{1}$ finite element package focusing on electromagnetic problems, even though recently the software was expanded to cover thermal and structural problems as well. The here presented method to incorporate anisotropic soft-iron properties was originally developed by VectorFields [2].

In Opera 3D $\mu_{r}$ for the various directions is set by an internal function and therefore varied at each iteration step. The data for $\mu_{r}$ is supplied by a table from a text file (interpolated); in this particular example two different functions are supplied for the preferred direction and perpendicular to this; the permeability is evaluated for the particular magnetic flux density for the volume element of the structure under consideration.

In principle it is also possible to set-up an interpolation table as a function of up to three variables, which would allow to vary $\mu_{r}$ as a function of the magnetic flux density and angle of the field with respect to the grain orientation.

The following excerpt of the comi-script shows the essential definitions:

\$CONSTANT \#Pack 0.98

\$FUNCTION 'GOSteelmur0.txt' MutH

\$FUNCTION 'GOSteelmur90.txt' MuoH

${ }^{1}$ Cobham CTS Limited, 24 Bankside, Kidlington, Oxfordshire, OX5 1JE, UK 




In this definition \#pack defines the packing factor, that is how much of defined iron yoke volume is occupied by iron and how much is other material (for example sheets of insulation).

GOSteelmur0.txt and GOSteelmur90.txt contain the data to be interpolated (in this case $\mu_{r}$ versus $B$ ) according to Opera's table format. As a first step it is sufficient to define tables with data for the direction tangential and orthogonal to the grain direction. $\mathrm{hlx}$, $\mathrm{hly}$ and $\mathrm{hlz}$ set the initial value of the magnetic field strength to zero, which is a required step for the initial initialization.

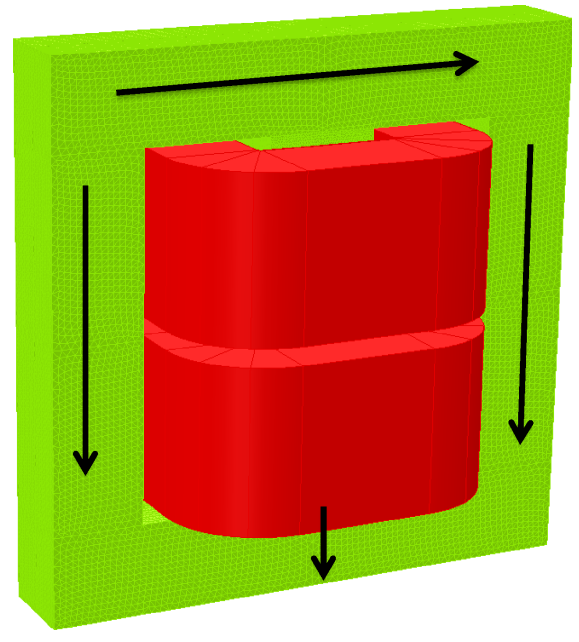

Figure 4: H-frame dipole. The direction of the grain orientation is indicated by the black arrows. Each of the areas needs to be a separate volume in Opera to adjust the local coordinate system to a suitable direction.

For the analysis it is assumed that the local z-coordinate is the direction of the grains in 
the soft-iron. The local coordinate system of various parts of the iron yoke (i.e. volumes) in Opera is adjusted so that the grain orientation aligns with the (local) z-direction.

The magnetic permeability for the $x, y$ and $z$ direction in Cartesian coordinates is defined by \#MuX, \#MuY and \#MuZ.

In the example under consideration parts of the iron yoke with different grain orientation need to be different subdomains, as indicated in Fig. 4.

For this analysis the material properties in grain direction $\left(0^{\circ}\right)$ and perpendicular to that are considered $\left(90^{\circ}\right)$, which should be a worst case. To estimate the effect of the varying $\mu_{r}$ with angle to the grain direction either a sensitivity study can be carried out (repeating simulations with a $\mu_{r}$ for a different angle) or a function of two variables (field and angle) can be used.

Opera 2D was also employed, which in difference to Opera 3D solves for the magnetic vector potential (Opera 3D solves for the scalar potential). In Opera 2D different BHcurves can be set for the $x / y$-direction.

\section{Geometry}

The simulations aimed to explain the behaviour of the fabricated test magnets at the University of Mississippi, Oxford, MS, USA. A schematic of the first magnet is shown in Fig. 5 (a); a second magnet using mitre-joints was simulated as well (see Fig. 5 (b)).
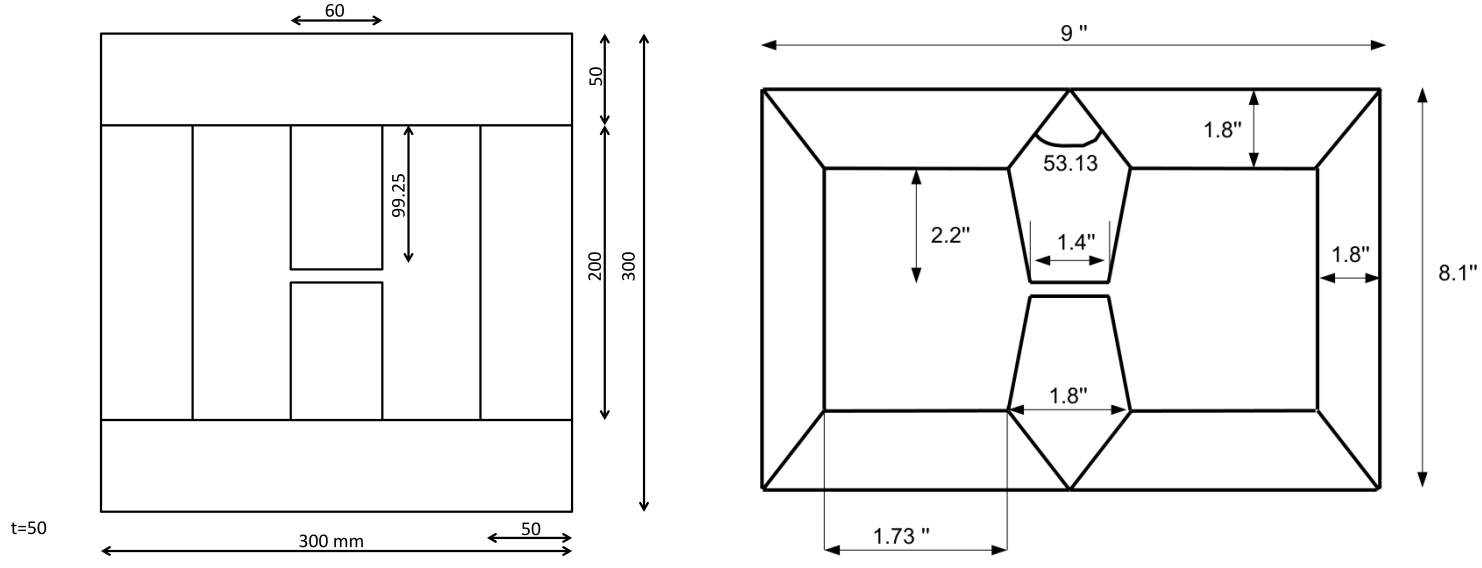

Figure 5: Geometries of the simulated magnets. Fig. (a) shows the first manufactured magnet using butt-joints, whereas Fig. (b) employs mitre-joints.

For the 3D simulations different slab thicknesses were simulated, from $<1 \mathrm{~mm}$ to $50 \mathrm{~mm}$. 


\section{Simulation Results}

It was found that a major challenge when modelling grain oriented steel is the convergence of the simulations; this applies to all simulation packages under consideration. During the evaluation it was not possible to obtain simulation results accurate enough for the desired purpose.
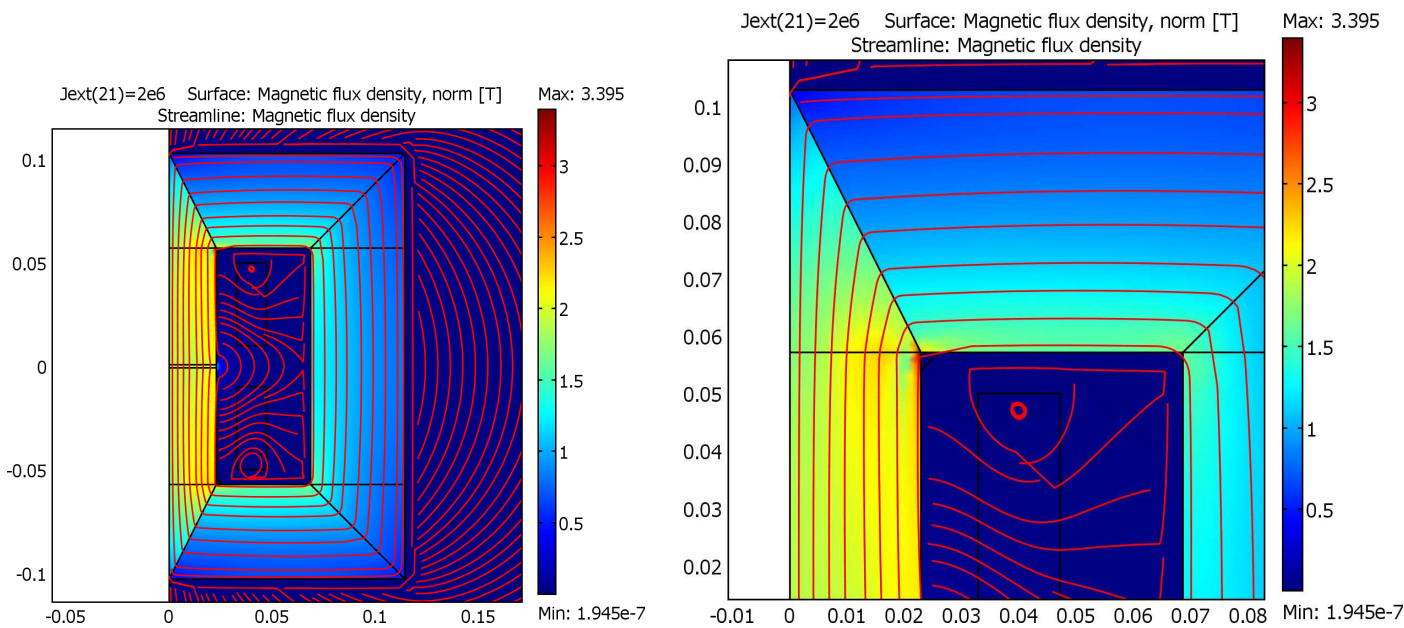

Figure 6: COMSOL simulation result of the mitre geometry; the simulation result indicates that regions of high magnetization exist right next to regions of low magnetization, which is non-physical.

It was established that the convergence problems are directly linked to the anisotropic material properties of the steel. In detail, the major complication seems to be the different shape of the BH curves in different directions; the simulations converged when just scaling one of the existing $\mathrm{BH}$ curves (for example, for $B$ perpendicular to the grain direction $\mu_{r}$ is $5 \%$ of $\mu_{r}$ parallel to the grain direction).

The following list gives a short overview of attempts to resolve this.

Software packages: A number of commercial and free software packages were tried, including Opera 2D/3D and COMSOL. Other software packages were considered (Poisson Superfish ${ }^{2}, \mathrm{FEMM}^{3}, \mathrm{MATLAB}^{4}$ ), but dismissed due to lacking 3D capability. The experienced problems and performance was similar in comparison to Opera/COMSOL and the preliminary studies are omitted here.

Mesh size: Convergence could not be improved by decreasing the mesh size.

\footnotetext{
${ }^{2}$ http://laacg1.lanl.gov/laacg/services

${ }^{3}$ http://www.femm.info

${ }^{4}$ http://www.mathworks.com/
} 

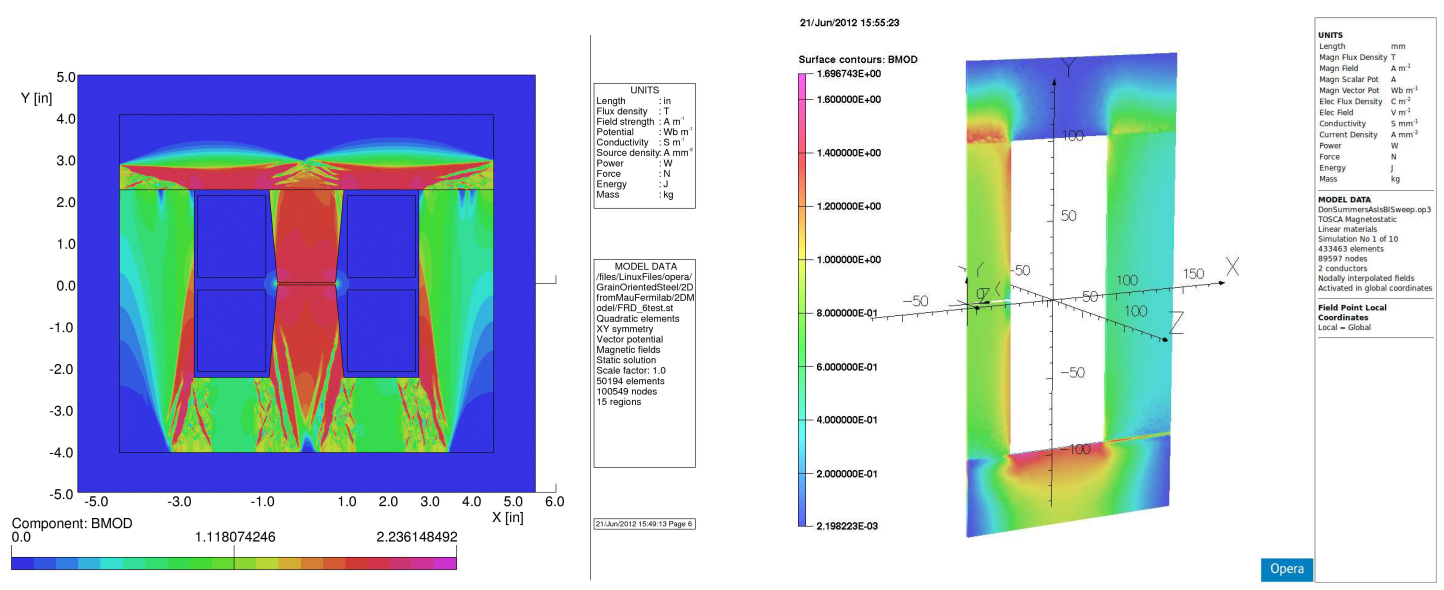

Figure 7: Opera 2D simulation result of the mitre geometry (Fig. (a), courtesy of Mauricio de Lima Lopes, FNAL) and Opera 3D simulation result of the butt-joint magnet (b). Both simulations did not converge.

Geometry: In addition to the butt-joint and mitre design additional, simplified geometries were tried. For example, the laminate thickness was increased to $50 \mathrm{~mm}$ to rule out any problems due to the thin lamination. Air gaps were introduced between joints to improve convergence, without any success. Single laminates were modelled as well as stacks of opposite ones.

BH curves: The smoothness of the BH curves was improved by adding more points or by using more suitable interpolation functions. For Opera 3D, BH curves were included in different ways (method described earlier and using the GUI, which allows to set different $\mathrm{BH}$ curves for different directions in each volume) to rule out any interface problems. In COMSOL the BH curve was included as a look-up table (linear/bilinear interpolation) and an analytic expression.

Different BH-curves were tried to rule out any particular problem with grain oriented steel. The models also did not converge when using two pre-supplied but different BH-curves in Opera (e.g. 'Default' and 'Armco Smith'). Fig. 8 (b) shows the result of this attempt.

Re-implementation of the Magnetostatics Application Mode: To rule out any problems with the pre-programmed application mode used in COMSOL for Magnetostatic problems the PDE was re-implemented. The PDE $\left(\nabla\left(-\frac{1}{\mu_{0} \mu_{r}} \nabla A_{z}\right)=J_{\text {ext }}\right)$ was implemented in the so-called coefficient and general form. Both re-implementations suffered from the same convergence issues.

Solvers: In COMSOL a number of different solvers were tried. This included direct solvers 
$\left(\right.$ UMFPACK $^{5}$, SPOOLES ${ }^{6}$, PARDISO ${ }^{7}$ ) as well as indirect solvers (GMRES, CG, Geometric Multigrid).

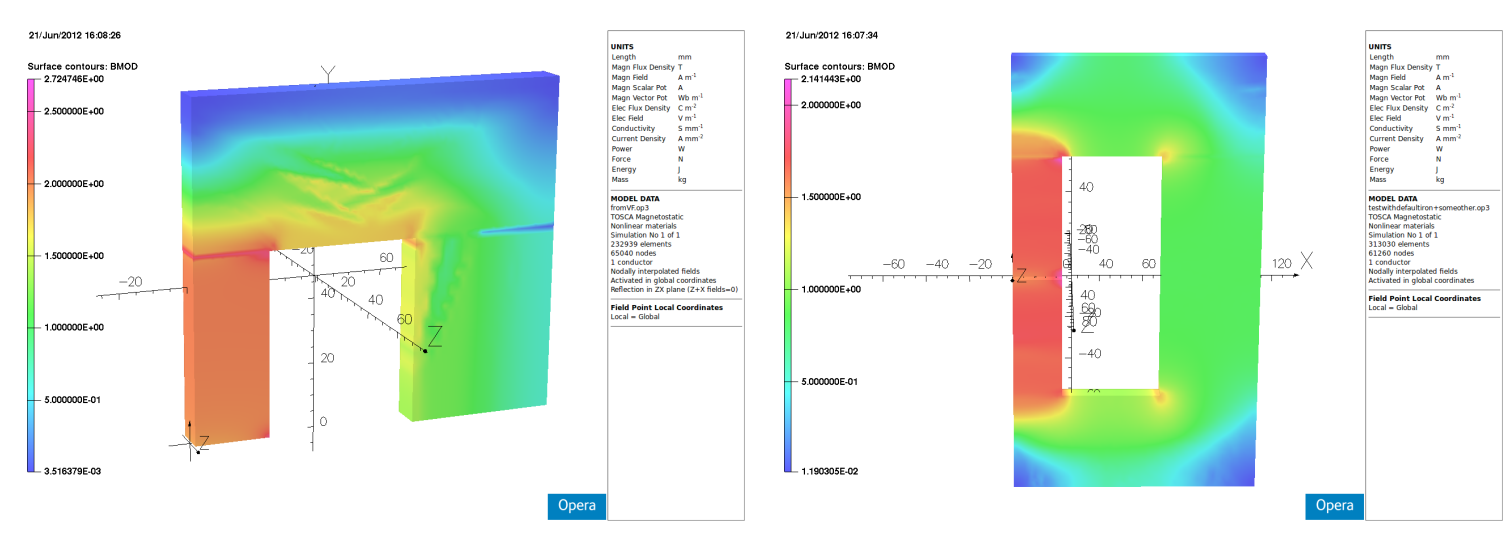

Figure 8: Opera 3D simulation result of the magnet with mitre joints using grain-oriented steel (a) and two of the pre-supplied BH curves (Fig. (b), 'Default' and 'Armco Smith').

Despite all efforts convergence could not be improved; neither the COMSOL nor the Opera 2D/3D simulations converged. The results showed non-physical behaviour in that all simulations predicted regions of very high magnetization (close to saturation) right next to areas with no or little magnetization. Several examples of this are shown in Fig. 6, 7 and 8.

\section{Summary}

This report summarizes efforts to include grain oriented steel in finite element simulations to model a dipole accelerator magnet. The report focuses on two commercially available packages: COMSOL and Opera 2D/3D from Vectorfields.

In principle both software packages should allow to include anisotropic non-linear magnetization curves. In practise it was found that convergence problems limit this feature considerably, making it unsuitable to model accelerator magnets due to the lack of accuracy.

A further problem arises in that none of the codes under consideration can estimate core losses of anisotropic material accurately. While all codes can estimate eddy currents, the modelling of hysteresis losses is limited to isotropic materials (Opera).

Preliminary simulation results suggest that the magnetic flux follows strongly the direction of the grains in the material, which is to be expected from the BH-data. This could

\footnotetext{
${ }^{5}$ http://www.cise.ufl.edu/research/sparse/umfpack/

${ }^{6} \mathrm{http}: / /$ www.netlib.org/linalg/spooles/spooles.2.2.html

${ }^{7} \mathrm{http}: / /$ www.pardiso-project.org/
} 
imply a relatively low tolerance against material faults and manufacturing tolerances, as for magnetizations close to saturation the material behaves as a $1 \mathrm{D}$-material. This may jeopardize if not rule out the use of anisotropic materials for accelerator magnets. Further concerns are long-term stability and resistance to radiation, which are not addressed in this report.

In summary, to study the potential benefits and shortcomings of Grain Oriented Steel for accelerator magnets a significant effort has to be made to understand the nature of the convergence problems and the subsequent improvement of one of the available finite element codes. 


\section{References}

[1] AK Steel. Oriented \& TRAN-COR H ELECTRICAL STEELS. Product Data Bulletin, AK Steel, AK Steel Corporation, 9227 Centre Pointe Drive, West Chester, Ohio, 45069, USA, 2011.

[2] E. Moor. Private communication. Technical Support, Vector Fields Software, Cobham Technical Services, 24 Bankside, Kidlington, Oxford OX5 1JE, UK, September 2011.

[3] D. Summers. Fast Ramping $750 \mathrm{GeV}$ Muon Synchrotron. Presentation at Muon Acceleration Program, Muon Collider 2011: Physics - Detectors - Accelerators, June 2011. Telluride, Colorado, USA.

[4] D. Summers, L. Cremaldi, R. Godang, B. Kipapa, H. Rice, et al. Muon acceleration to $750-\mathrm{GeV}$ in the Tevatron tunnel for a $1.5-\mathrm{TeV} \mathrm{mu}+\mathrm{mu}-$ collider. Conf.Proc., C070625:3178, 2007. 